S. A. Prahl and S. L. Jacques, "Sized-Fiber Array Spectroscopy," in Laser-Tissue Interaction IX, S. L. Jacques Ed., Proc. SPIE 3254, 348-352, (1998).

\title{
Sized-Fiber Array Spectroscopy
}

\author{
S. A. Prahl, S. L. Jacques \\ Oregon Medical Laser Center, 9205 SW Barnes Rd, Portland, OR 97225
}

\begin{abstract}
Sized-fiber array spectroscopy describes a device and method for measuring absorption and reduced scattering properties of tissue. The device consists of two or more optical fibers with different diameters (comparable to the optical path length in the tissue) that are used to measure the amount of light backscattered into each fiber. Each fiber is used for both irradiation and detection. Only one fiber emits and collects light at a given time. This paper presents Monte Carlo simulations of the sized-fiber device to indicate the behavior of a device with 50 and $1000 \mu \mathrm{m}$ fiber sizes. Experimental results are presented for a device constructed with 400 and a $600 \mu \mathrm{m}$ fibers that demonstrate the accuracy of the device in measuring the scattering coefficient of $10 \%$-Intralipid samples over a reduced scattering coefficient range of $1-50 \mathrm{~cm}^{-1}$.
\end{abstract}

Keywords: Reflectance, Optical Biopsy

\section{INTRODUCTION}

The determination of the optical properties of materials is important in many fields of medicine. The sized-fiber device described in this paper allows simple and rapid measurement of optical properties by taking advantage of the fact that the amount of light backscattered into an optical fiber is affected by the scattering and absorption properties of the tissue. Furthermore, the sized-fiber array satisfies the medical demands that a clinical device to determine the optical properties of tissue be simple to construct, inexpensive to build, compact in size, and robust in operation.

The sized-fiber device is based on the fact that, generally, tissues with different scattering and absorbing properties will backscatter different numbers of photons into the originating fiber. However, it is possible for two samples (say $\mathrm{A}$ and B) with very different optical properties to backscatter the same number of photons back into a particular size fiber, and a single measurement with one fiber would be insufficient to distinguish the two samples from each other. If a measurement is made on samples $\mathrm{A}$ and $\mathrm{B}$ with a second fiber, having a different diameter from the first, then the two sample will be distinguishable. This is because different sized fibers collect information from different effective volumes of the samples.

This paper begins with Monte Carlo simulations to support this claim. Simulations for a device consisting of a 50 and $1000 \mu \mathrm{m}$ fiber are presented. This is followed by a description of an experiment to measure the scattering properties of Intralipid. The experimental results indicate that the method is sensitive to changes in reduced scattering from 1 to $50 \mathrm{~cm}^{-1}$.

\section{MONTE CARLO SIMULATIONS}

A Monte Carlo program was adapted to simulate the light collected by a fiber irradiating a homogenous scattering and absorbing medium. ${ }^{?, ?}$ A series of simulations were done to demonstrate the feasibility of the sized-fiber method for measuring the optical properties of materials and to interpret experimental the measurements in Intralipid.

Let the axis of the fiber be parallel to the $z$-axis, and further let this axis be normal to the surface of a semi-infinite scattering and absorbing medium. The face of the fiber is flush against the surface of the medium. The illumination over the face of the fiber is uniform i.e., the photons are launched with equal probability over the entire face of the fiber.

Photons were launched from each point on the fiber face in a direction specified by the direction cosines $\left(\nu_{x}, \nu_{y}, \nu_{z}\right)$. The angle $\nu_{z}=\cos \theta_{z}$ had a Gaussian distribution that depended on the acceptance angle of the fiber and the other

Direct correspondence to S.A.P, prahl@ece.ogi.edu; (503) 216-2197; http://omlc.ogi.edu 


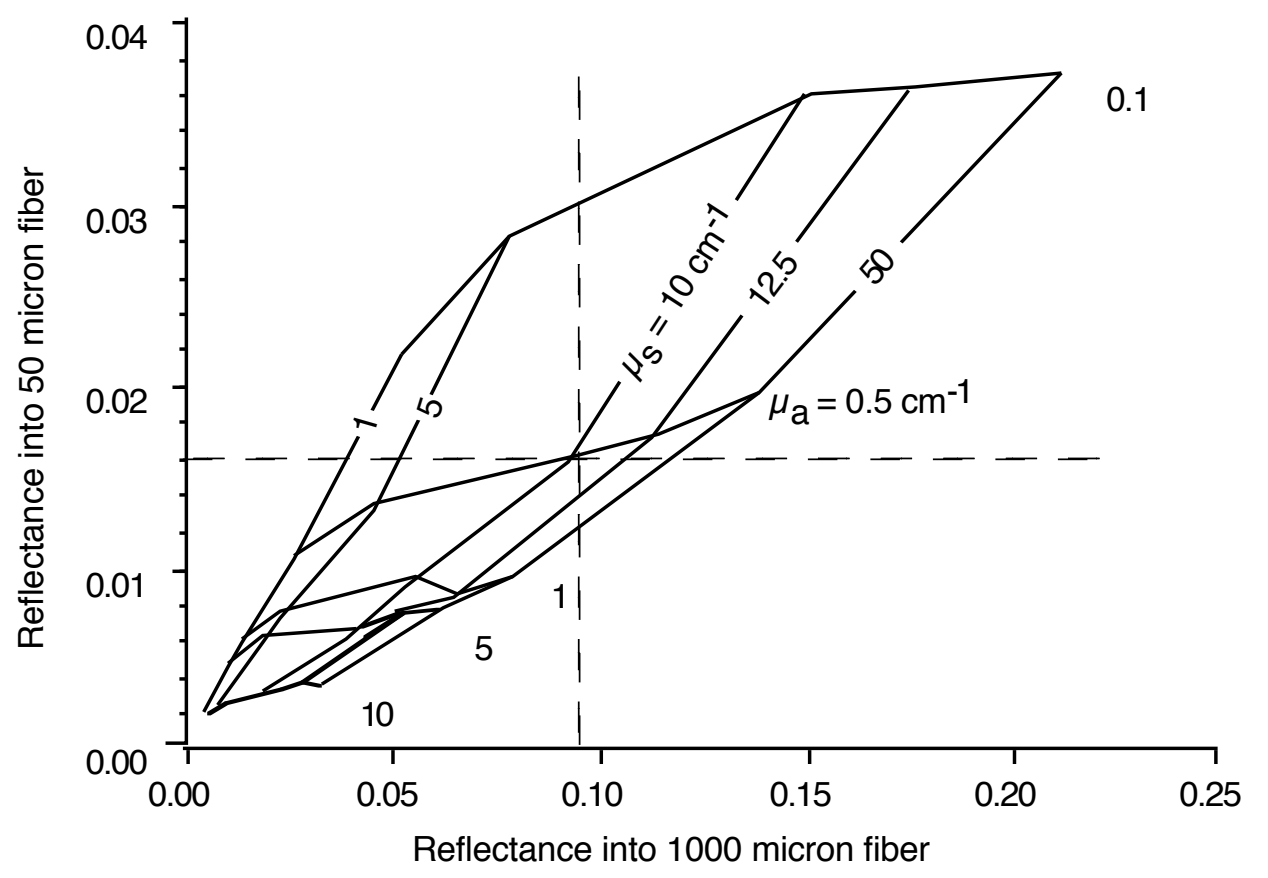

Figure 1. The fraction of light collected by a $50 \mu \mathrm{m}$ and $1000 \mu \mathrm{m}$ fiber for various combinations of optical properties. The reduced scattering coefficient is $\mu_{s}$ and the absorption coefficient is $\mu_{a}$. From this graph it is evident that for a specific reflectance from a $50 \mu \mathrm{m}$ fiber and from a $1000 \mu \mathrm{m}$ fiber, a specific optical property is obtained. For example, if $R_{50}=0.016$ and $R_{1000}=0.09$ then $\mu_{a}=0.05 \mathrm{~cm}^{-1}$ and $\mu_{s}^{\prime}=10 \mathrm{~cm}^{-1}$.

two directions were uniformly distributed. This angular distribution was chosen to simulate the major features of the observed emission by the fibers; it was not directly confirmed experimentally. The distribution of angles that the photon might take was given by the function

$$
p\left(\theta_{z}\right) d \theta_{z}=\frac{1}{\sqrt{2 \pi}} \exp \left(-\frac{\theta_{z}^{2}}{2 \theta_{a}^{2}}\right) d \theta_{z}
$$

where $\theta_{a}$ was the acceptance angle for the fiber. Furthermore, to limit the angles on the shoulders of the distribution, only those values of $\theta_{z} \leq \theta_{a}$ were accepted. The other two angles were generated using

$$
\nu_{x}=\left(1-\nu_{z}^{2}\right) \cos \phi \quad \text { and } \quad \nu_{y}=\left(1-\nu_{z}^{2}\right) \sin \phi
$$

where $\phi=2 \pi \xi$ was an azimuthal angle about the $z$-axis and $\xi$ is a uniformly distributed random number between zero and one. This preserved the all important relation

$$
\nu_{x}^{2}+\nu_{y}^{2}+\nu_{z}^{2}=1
$$

The primary statistic collected by the Monte Carlo program was the fraction of light backscattered or reflected back into the fiber. For photons to be counted as reflected they must have exited the surface at a radius that was within the core of the fiber and at an angle that was within the acceptance angle of the fiber. The index of refraction of the core of the fiber was assumed to be 1.46 and the index of the medium was 1.38. Scattering in the medium was assumed isotropic. A total of 20,000 photons were used for each data point.

Figure ?? shows the results of Monte Carlo calculations for a range of optical properties and for a 50 and $1000 \mu \mathrm{m}$ fiber. The acceptance angle of the fibers were assumed to be $25^{\circ}$ in air. As scattering increases from 1 to $50 \mathrm{~cm}^{-1}$, the reflectances into both fibers increase; although less so for the smaller fiber. As the absorption decreases, both reflectances increase, but the effect upon the larger fiber is smaller. 


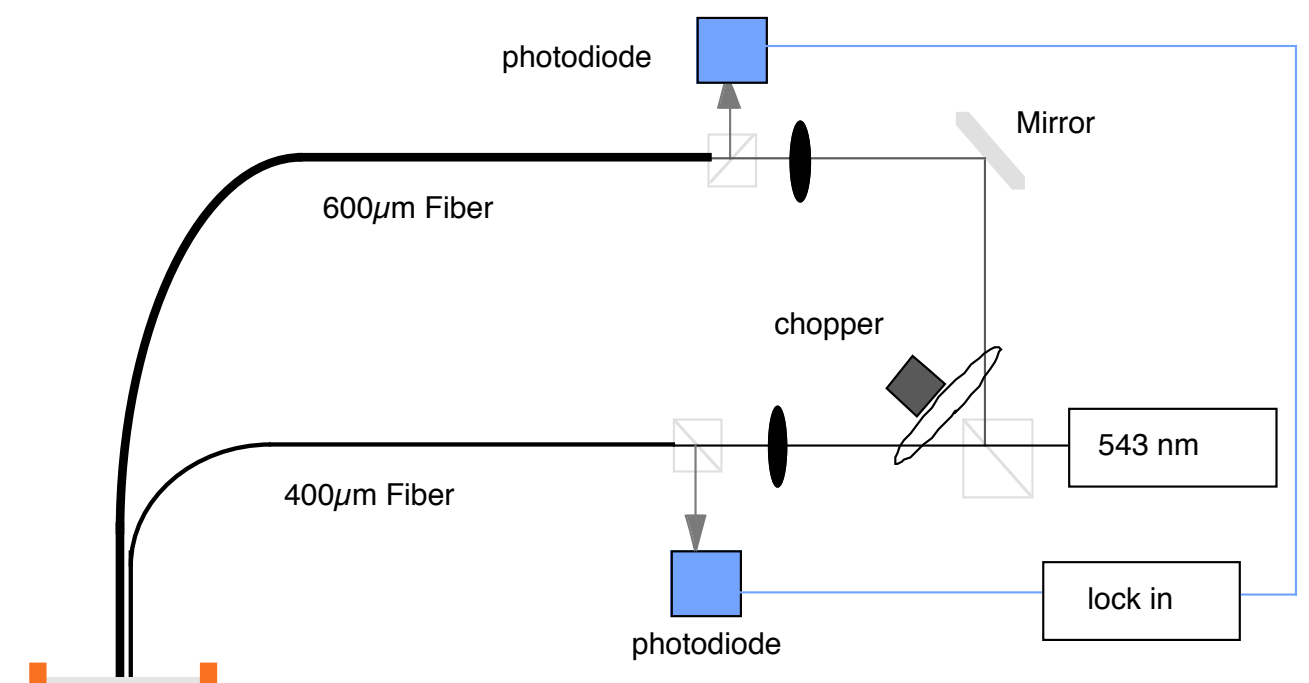

$10 \times 10 \times 10 \mathrm{~cm}$

Figure 2. The experimental apparatus used to test the sized-fiber device. Light from the green HeNe is coupled into two fibers with different sizes. Light only travels through one fiber at a time because of the orientation of the chopper blade. The light backscattered into the fibers is detected with large photodiodes and a lock-in amplifier.

\section{MATERIALS AND METHODS}

The experimental apparatus is shown in Figure ??. The beam from a green He-Ne laser operating at $543 \mathrm{~nm}$ was split into two beams with a non-polarizing $50 / 50$ beam splitter. Both beams were modulated at $1 \mathrm{kHz}$ by a single chopper blade (Stanford Research, SR540) in such a way that only one beam was on at a particular instant. Each beam passed through a second beam splitter and were coupled into a $400 \mu \mathrm{m}$ and a $600 \mu \mathrm{m}$ fiber with acceptance angles in air of 13 and $25^{\circ}$ respectively. The distal fiber tips were completely immersed in the Intralipid solutions; this is different from the Monte Carlo simulations, but seemed to make relatively little difference in the measured signals. The light backscattered into the fiber was divided again by the beam splitter and half was collected with a photodiode. The outputs from the photodiodes and chopper were coupled into a lock-in amplifier (Stanford Research, SR510)

The samples measured were dilutions of Intralipid with optical properties from 1 to $50 \mathrm{~cm}^{-1}$. No absorber was added and consequently the absorption coefficient was assumed to be a nominal $\mu_{a}=0.01 \mathrm{~cm}^{-1}$.

\section{RESULTS}

The experimental results for the Intralipid experiments are shown in top graph of Figure ??. In this graph the theoretical Monte Carlo simulation is shown as the solid line. It is not quite straight because only 20,000 photons were used. The numbers beside the line indicate the reduced scattering coefficient for the square nearest the number. The error bars on the measured data are the standard deviation of five measurements.

The graph in the bottom of Figure ?? shows how the measured values taken from top graph compare with the expected values for the various dilutions of Intralipid. The measured values were taken as the nearest point on the theoretical line in the top graph. 

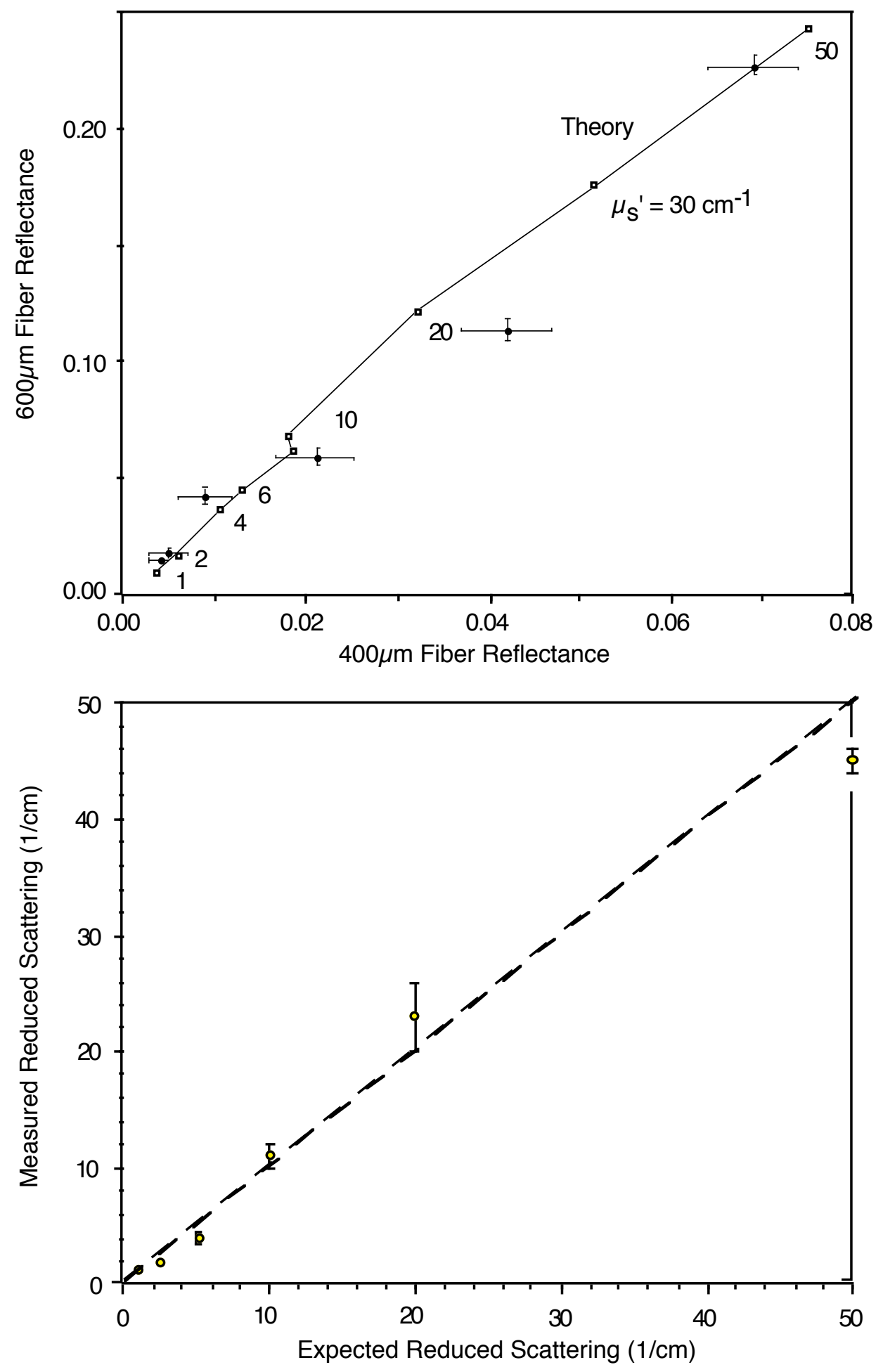

Figure 3. (Top) The fractions of light collected by 400 and $600 \mu \mathrm{m}$ fibers in various concentrations of Intralipid. (Bottom) A comparison of the expected reduced scattering coefficient with the reduced scattering coefficient determined with the sized fiber device. The dashed line indicates equal values for the measured and expected values of the reduced scattering coefficient. 


\section{DISCUSSION}

The sized-fiber technique is yet another method for measuring the optical properties of materials.?,?,? This particular technique has the advantage that it can be made very small. The accuracy and sensitivity depend on choosing the proper fiber sizes for the material being probed.

What are the limitations of the sized fiber technique? First, scattering must be present in the sample or no light will be backscattered into the fibers. Consequently, the sized-fiber device will not work with absorbing-only media. Second, if the absorption in the sample is very high, then the returned signal becomes very small and the technique works less well. Third, the sizes of the fibers should be comparable to the average reduced scattering pathlength in the sample. Fourth, the conversion of measured values to optical properties remains to be reduced to a simple algorithm.

What are the limitations of this study? The experimental results only determine the scattering coefficients for an assumed absorption coefficient. It remains to be seen how much measurement errors will cause crosstalk in the signals for scattering and absorption. Finally, the choice of fibers for the experiment was poor - the fibers were much too close in size to one another.

What are the advantages to the sized-fiber technique? First, the optical properties are measured over a relatively small area. This is a definite advantage over the measurements made as a function of radius ${ }^{?}$ or the oblique incidence technique. ${ }^{?}$ Second, this technique can be naturally incorporated into time-domain techniques ${ }^{?}$ or into frequency-

domain techniques. ${ }^{?}$ Another advantage is that the sized-fiber technique are that it can be adapted to very small fibers for possible insertion through a needle. Alternately, the device could be used into a catheter for probing arterial structures or blood flow or into an endoscope for investigating gastro-intestinal structures.

\section{Acknowledgments}

This work was supported by a grant from the Department of the Army, Grant No. DAMD17-96-1-6006 and does not necessarily reflect the position or the policy of the government. No official endorsement should be inferred.

\section{REFERENCES}

1. A. N. Witt, "Multiple scattering in reflection nebulae I. A Monte Carlo approach," Astrophys. J. S35, pp. 1-6, 1977.

2. S. A. Prahl, M. Keijzer, S. L. Jacques, and A. J. Welch, "A Monte Carlo model of light propagation in tissue," in SPIE Proceedings of Dosimetry of Laser Radiation in Medicine and Biology, G. J. Müller and D. H. Sliney, eds., vol. IS 5, pp. 102-111, 1989. monte carlo.

3. H. J. van Staveren, C. J. M. Moes, J. van Marle, S. A. Prahl, and M. J. C. van Gemert, "Light scattering in Intralipid-10\% in the wavelength range of 400-1100 nm," Appl. Opt. 31, pp. 4507-4514, 1991.

4. B. C. Wilson, M. S. Patterson, and S. T. Flock, "Indirect versus direct techniques for the measurement of the optical properties of tissues," Photochem. Photobiol. 46, pp. 601-608, 1987.

5. W. F. Cheong, S. A. Prahl, A. J. Welch, M. J. C. van Gemert, and C. R. Denham, "Optical properties of bladder tissue and optimal dosage predictions for photoradiation therapy," Lasers Surg. Med. 6, pp. 190-191, 1986.

6. A. J. Welch and M. J. C. van Gemert, Optical-Thermal Response of Laser Irradiated Tissue, Plenum Press, 1995.

7. B. C. Wilson, T. J. Farrell, and M. S. Patterson, "An optical fiber-based diffuse reflectance spectrometer for non-invasive investigation of photodynamic sensitizers in vivo," in SPIE Proceedings of Future Directions and Applications in Photodynamic Therapy, C. J. Gomer, ed., vol. IS 6, pp. 219-232, 1990.

8. L. Wang and S. L. Jacques, "Use of laser beam with an oblique angle of incidence to measure the reduced scattering coefficient of a turbid medium," Appl. Opt. 34, pp. 2362-2366, 1995.

9. S. L. Jacques, "Time resolved propagation of ultrashort laser pulses within turbid tissues," Appl. Opt. 28, pp. 2223-2229, 1989.

10. S. Fantini, M. A. Franceschini, J. B. Fishkin, B. Barbieri, and E. Gratton, "Quantitative determination of the absorption spectra in strongly scattering media: a light-emitting-diode based technique," Appl. Opt. 33, pp. 5204-5213, 1994. 